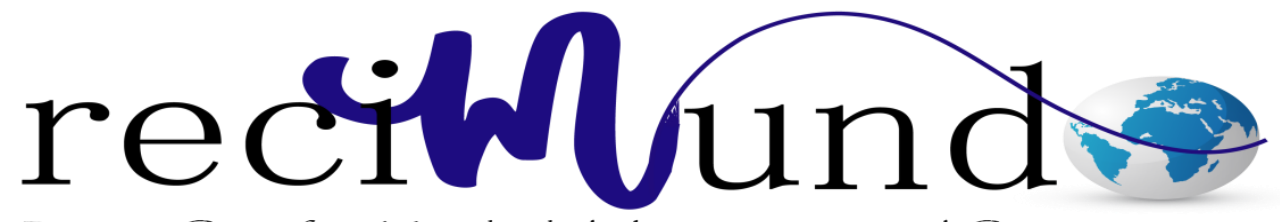

Revista Cientifica Mundo de la Investigación y el Conocimiento

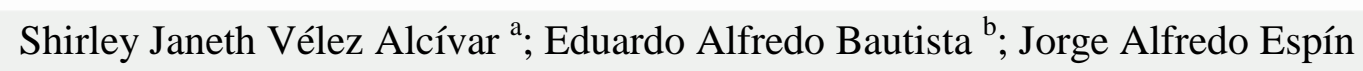
Martínez $^{\mathrm{c}}$

Didáctica Crítica de la Matemática Financiera

Revista Científica Mundo de la Investigación y el Conocimiento. Vol. 2 núm.2, mayo, ISSN: 2588-073X, 2018, pp. 552-565

DOI: 10.26820/recimundo/2.(2).2018.552-565

Editorial Saberes del Conocimiento

Recibido: 05/12/2017

Aceptado: 06/04/2018
a. $\quad$ shierleyvelezt@gmail.com
b. Universidad de Guayaquil; eduardo.bautistaq@ug.edu.ec
c. Universidad de Guayaquil; jorge.espinm@ug.edu.ec 


\section{Didáctica Crítica de la Matemática Financiera}

Vol. 2, núm. 2., (2018)

Shirley Janeth Vélez Alcívar; Eduardo Alfredo Bautista; Jorge Alfredo Espín Martínez

\section{RESUMEN}

La matemática financiera es de aplicación práctica, en operaciones, que relacionan capital, tasa de interés y tiempo, las firmas comerciales la usan para calcular los montos que deben cobrar al final del periodo pactado en cuotas, de modo que cuanto mayor sea el número de cuotas, mayor será la tasa de interés. Hoy día se aplica la matemática financiera en las actividades públicas y privadas, en el sistema bancario y no bancario, y se usa para el sistema de capitalización simple, compuesto y para la toma de decisiones. En este sentido, se da especial relevancia a la didáctica crítica en la enseñanza de la matemática como objeto de pensamiento crítico que propicie la transformación de los contenidos en relación a las necesidades del que aprende. Puede ser denominada didáctica distintiva, con la cual pueden crearse vínculos entre los sujetos del proceso enseñanza-aprendizaje, que a su vez, destaque la necesidad de sensibilizar a los docentes para que generen acciones educativas con bases teóricas hacia el conocimiento e intereses humanos.

Palabras clave: Didáctica, didáctica de la matemática, matemática financiera. 


\title{
Didáctica Crítica de la Matemática Financiera
}

Vol. 2, núm. 2., (2018)

Shirley Janeth Vélez Alcívar; Eduardo Alfredo Bautista; Jorge Alfredo Espín Martínez

\begin{abstract}
The financial mathematics is of practical application, in operations, that relate capital, interest rate and time, the commercial firms use it to calculate the amounts that must be collected at the end of the period agreed in installments, so that the higher the number of fees, the higher the interest rate. Nowadays, financial mathematics is applied in public and private activities, in the banking and non-banking system, and it are used for the simple, compound capitalization system and for decision-making. In this sense, special importance is given to critical didactics in the teaching of mathematics as an object of critical thinking that promotes the transformation of contents in relation to the needs of the learner. It can be called distinctive didactics, with which links can be created between the subjects of the teaching-learning process, which, in turn, highlights the need to sensitize teachers so that they generate educational actions with theoretical bases towards knowledge and human interests.
\end{abstract}

Key words: Didactics, mathematics didactics, financial mathematics. 


\section{Didáctica Crítica de la Matemática Financiera}

Vol. 2, núm. 2., (2018)

Shirley Janeth Vélez Alcívar; Eduardo Alfredo Bautista; Jorge Alfredo Espín Martínez

\section{Introducción.}

García (2012) expresa que las Matemáticas Financieras constituyen una disciplina que tiene por objeto el estudio de operaciones financieras; donde la actividad financiera surge paralelamente a la economía monetaria, en la que el dinero es unidad de cuenta, medio de pago o instrumento de cambio, depósito de valor o activo financiero, y en la que los bienes económicos son expresados en función de dos magnitudes, capital financiero, medido en unidades monetarias, y tiempo o momento en el que se puede disponer del mismo; de esta manera, el intercambio de bienes económicos así entendido da lugar a la aparición de los fenómenos y las operaciones financieras

La Matemática Financiera como rama de la matemática aplicada, que no se basa en la existencia de leyes regidas, absolutas. Son un conjunto de herramientas matemáticas, las cuales permiten analizar cuantitativamente la viabilidad o factibilidad económica y financiera de los proyectos de inversión. En este sentido, en las operaciones financieras intervienen los hombres que deciden qué operación realizar, cómo y cuándo, deciden sobre qué ley financiera van a realizar sus operaciones, de manera que los agentes económicos participantes salgan satisfechos.

Tales características, viables para la enseñanza y aprendizaje de la misma. Pero que con el transcurrir del tiempo, el docente la ha destacado como una simple herramienta de carácter complejo y netamente complicada en su enseñanza-aprendizaje. Marcando lo objetivo, lo medible y lo cuantificable, originando pasividad en el estudiante y completo dominio de experto. 


\section{Didáctica Crítica de la Matemática Financiera}

Vol. 2, núm. 2., (2018)

Shirley Janeth Vélez Alcívar; Eduardo Alfredo Bautista; Jorge Alfredo Espín Martínez

Si se desea una práctica educativa diferente a la impartida en los últimos dos siglos, entonces necesitamos romper, en gran medida, con la forma de presentar y trabajar el conocimiento matemático, dando paso a la ejecución de una didáctica acorde con las necesidades del estudiante, basados en el dialogo, la participación y la cooperación entre los integrantes de la práctica educativa.

En el presente artículo, se dispone una breve reflexión sobre la didáctica crítica de la matemática financiera, que ayude a comprender el papel de la misma dentro de la sociedad, no sólo como un cúmulo de formalizaciones materializadas, sino como tales formalizaciones aportan a alguna competencia ética. Revisar y profundizar en los apartados dispuestos: Educación y Educación Crítica, Didáctica de la Matemática y Didáctica Crítica de la Matemática Financiera. Nos llevará como docentes a la aplicación de cada concepto a temas cotidianos para una mejor comprensión de la esencia de lo que se quiere enseñar.

\section{Educación y Educación Crítica}

En el transcurrir de la historia moderna, la definición de educación se ha desarrollado hasta ser considerada como "un proceso de acomodar el futuro al pasado... una utilización del pasado como un recurso para desarrollar el futuro" (Dewey, 2004, p. 76). Igualmente, Vadillo y Klingler (2004) citan a Giner, expresando que la educación:

Es una acción universal, difusa y continua de la sociedad (y aun del medio todo), dentro de la cual la acción del educador intencional desempeña la función reflexiva, definida, discreta, propia del arte en los demás órdenes de la vida, de excitar la reacción personal de cada individuo y, aun de cada grupo social, para 


\section{Didáctica Crítica de la Matemática Financiera}

Vol. 2, núm. 2., (2018)

Shirley Janeth Vélez Alcívar; Eduardo Alfredo Bautista; Jorge Alfredo Espín Martínez

su propia formación y cultivo; todo ello mediante el educando mismo y lo que él de suyo pone para esta obra, ya lo ponga espontáneamente, ya en forma de una colaboración también intencional. (p. 2)

En el discurso desarrollado, estos pensadores han considerado a la educación como la base del progreso y del fundamento mismo del desarrollo, es decir, la estiman como el eje de la evolución social, siendo un proceso de formación integral que debe potenciar las actitudes de toda persona, tanto como individuo como por ser miembro de una sociedad solidaria, debe consustanciarse con la evolución social ya que conduce a la comprensión de la persona, en sí mismo y de su entorno, basado en los conocimientos, experiencias y valores culturales que le son propios y compartidos en su proceso de socialización.

Cabe considerar la perspectiva teórica de Vygotsky (citado en Ramos, 2001), cuando plantea que:

La educación tiene la gran tarea socializadora de formar la conciencia. Se debe hallar un vínculo entre la cultura académica y la cultura vulgar. Los contenidos disciplinarios no pueden ser otras herramientas, sino aquellas que sirven para reconstruir críticamente los propios significados y comportamientos. (p. 185)

Con base en lo referido, puede afirmarse que la formación de la persona es un simple contacto académico-social consigo mismo y su entorno, expresado a través del lenguaje, utilizando, además, los contenidos programáticos, quienes permiten la reconstrucción, de manera crítica, de sus propias ideas, que por ende, modifican su comportamiento. 


\section{Didáctica Crítica de la Matemática Financiera}

Vol. 2, núm. 2., (2018)

Shirley Janeth Vélez Alcívar; Eduardo Alfredo Bautista; Jorge Alfredo Espín Martínez

Teniendo como referencia la definición de pedagogía crítica que nos aporta Alvarado (20012), quien dice que se trata de una vertiente de la educación cuya finalidad es la de cuestionar las relaciones de poder establecidas por la sociedad, basadas en jerarquizaciones injustas y asimétricas. Y más allá, y nos plantea este tipo de educación como la que rompe con los patrones tradicionales, para proponer un modelo educativo multidireccional, donde desplaza las jerarquizaciones injustas y subraya el trabajo de todos los agentes que intervienen en la educación dentro de un proceso participativo y democrático. Así pues, hablar de pedagogía crítica, es referirse a la vinculación de la educación con la justicia social.

Para Santaella (2011), uno de los autores más relevantes en este campo, y cuyas teorías pedagógicas han servido como referente a todos los defensores de la pedagogía crítica, es Paulo Freire. Él nos habla de deshumanización y humanización, el primer concepto hace referencia a una actividad de dominación, cuya finalidad es la de conservar el statu quo, muestra una visión de la realidad estática imposible de cambiar más que por las clases dominadoras en función de sus propios intereses; el segundo viene a hacer mención al proyecto de cambio que se debe poner en marcha por parte de las clases dominadas, y por tanto más desaventajadas. Estos dos conceptos, aunque ambos suponen una acción de la sociedad sobre la realidad, hacen referencia a dos ideas totalmente opuestas, ya que una pretende el cambio de la realidad social y la otra conservar el estado vigente (Freire, 1978).

Con referencia a estas dos ideas Freire (1978) nos propone dos modelos educativos. En su libro Educación y acción liberadora, habla de educación dominadora por un lado, y de la educación liberadora por otro. La primera viene a hacer referencia a un modelo educativo que se ajusta a los objetivos referentes a la deshumanización, la cual trata de una educación 


\section{Didáctica Crítica de la Matemática Financiera}

Vol. 2, núm. 2., (2018)

Shirley Janeth Vélez Alcívar; Eduardo Alfredo Bautista; Jorge Alfredo Espín Martínez

unidireccional, en la que el educador realiza un trasvase de sus saberes sobre el educando, quedando fuera aspectos tales como el de generar una conciencia propia y libre sobre la realidad, como el autor afirma, se trata de una educación que tiene la obligación de dominar, se enseña lo que se "debe ser". Mientras que cuando habla de educación liberadora, o acción cultural liberadora, hace referencia a un modelo educativo basado en un acto puro de conocimiento, donde educandos y educadores construyen nuevas formas de conocimiento y de saber, como seres conscientes y activos del mundo en el que viven. En este sentido todos los hombres y mujeres forman parte de la realidad social, y por tanto pueden ser agentes activos del cambio de rumbo, hacia modelos más justos y democráticos, este modelo viene a hacer referencia a los principios por los que se rige la pedagogía crítica.

Si tentativamente tratamos de resumir en qué consiste la educación crítica, una idea simple podrá ser: si las prácticas y la investigación educativa son críticas, deben abordar los conflictos y las crisis en la sociedad. La educación crítica debe revelar las desigualdades y la represión de cualquier tipo. Una educación crítica no debe contribuir simplemente a la prolongación de las relaciones sociales existentes; no puede ser el medio para perpetuar las desigualdades existentes en la sociedad. Si desea ser critica, una educación debe reaccionar a la naturaleza crítica de la sociedad.

\section{Didáctica de la Matemática}

La didáctica, en términos de una teoría de la enseñanza, fue utilizada por el pedagogo alemán Wolfgang Ratke (1571-1635) (antes se usaba el concepto de arte de enseñar, dándole la acepción de técnica a la palabra arte). Pero fue Juan Amos Comenio (1592-1670) quien la dejó como palabra con categoría pedagógica cuando la usó como título en su Didáctica Magna. 


\section{Didáctica Crítica de la Matemática Financiera}

Vol. 2, núm. 2., (2018)

Shirley Janeth Vélez Alcívar; Eduardo Alfredo Bautista; Jorge Alfredo Espín Martínez

A medida que ha trascendido el concepto de didáctica, la pedagogía de todos los tiempos se ha visto comprometida en darle una definición, de allí, que sus constructos han sido basados en la experiencia pedagógica, por lo que a través de la historia, la didáctica es conocida como la “teoría general de la enseñanza" Valiente (2000), en donde se dan los contenidos de la enseñanza y sus características, modos, procedimientos, formas, actos y, por supuesto, los métodos, por lo cual ha llegado a ser dividida en: didáctica general y didáctica especial.

La didáctica general, hace referencia a disposiciones frecuentes como son: formas de la enseñanza, recursos de la enseñanza, objetivos, contenidos. Y la didáctica especial, es aquella que incide sobre la generalidad de todos los elementos anteriores, pero va a las particularidades de cada asignatura del conocimiento.

Para Ugas (2007), la pedagogía, al igual que la didáctica, presenta concepciones diferentes; ello, depende de la posición asumida por cada autor. Por ello, en adelante, se conoce a la pedagogía como la ciencia de la educación y/o el arte de educar. También, en su estudio, refiere que, el término pedagogía ha pasado a designar la práctica de los maestros de la escuela primaria a los que se les pasa a denominar -pedagogos-. "Ergo: pedagogía pasa a designar la acción de enseñar mas que al proceso de teorizar o reflexionar sobre sus fundamentos” (pg. 113).

Aunado a lo anterior para Ugas (ob. cit) el término didáctica designa el estudio de la relación alumno-profesor mediada por los conocimientos constituidos en disciplinas escolares; vale decir, designa la relación entre los contenidos enseñados, los educandos y el personal docente. Así surgen didácticas para enseñar: a) contenido para cada disciplina y la manera de presentar un ( $\mathrm{su}$ ) conocimiento; y, b) el estudio de la transmisión de conocimientos (teóricos y 


\section{Didáctica Crítica de la Matemática Financiera}

Vol. 2, núm. 2., (2018)

Shirley Janeth Vélez Alcívar; Eduardo Alfredo Bautista; Jorge Alfredo Espín Martínez

prácticos). De esta manera, la didáctica pasa a ser al mismo tiempo una teoría y una metodología. (Ugas, ob. cit.).

En consecuencia, se puede argumentar que hoy día, la didáctica representa el conjunto de normas, criterios, recursos y medios con los cuales la práctica docente se realiza. Es por ello que debe existir una correspondencia biunívoca entre la relación del sujeto que se dedica a enseñar y el que va a ser enseñado. Quien se dedica a enseñar, debe imprimir voluntad, actitud, energía y tiempo al rasgo de la actividad humana (enseñanza) que se desarrolla en el espacio y en el tiempo: en el espacio pedagógico y en el tiempo histórico-cultural. La persona que se desea formar, está sujeta a las determinantes de la sociedad, de la historia, de la política, de la economía, de la cultura toda. Así pues, dado estos aspectos, la educación es actividad concreta cuyo sustento ideológico está en la superestructura social y la determina la sociedad en su conjunto dadas las reglas del juego establecidas entre las clases sociales mediante normas constitucionales. Es importante acotar que, dado el carácter social de la educación en cuanto a la formación de ciudadanos, no asegura la incorporación de los individuos a las estructuras sociales.

Por otro lado Dámore (2006) acota que la didáctica es parte de las ciencias de la educación que tiene como objetivo el estudio de los procesos de enseñanza y aprendizaje en su globalidad independientemente de la disciplina en objeto, pero teniendo en cuenta la relación institucional; aunque muchos autores eliminarían la citación de relación institucional, pero darían en cambio más peso a las disciplinas. Ya que la disciplina en sí (si nos referimos a la matemática) referencia el cómo se le conoce y cómo es practicada por los especialistas, por los científicos, por parte de los matemáticos, en fin. 


\section{Didáctica Crítica de la Matemática Financiera}

Vol. 2, núm. 2., (2018)

Shirley Janeth Vélez Alcívar; Eduardo Alfredo Bautista; Jorge Alfredo Espín Martínez

Así pues, la didáctica de la matemática según Douady (1984) en DÀmore (2006), es el estudio de los procesos de transmisión y de adquisición de los diferentes contenidos de esta ciencia y se propone describir y explicar los fenómenos relativos a las relaciones entre su enseñanza y su aprendizaje. No se reduce a buscar una buena forma de enseñar una determinada noción. En este mismo orden, Vergnaud (1985) también en DÀmore (2006), la define como una disciplina que estudia los procesos de transmisión y de adquisición relativos al dominio específico de la matemática, o de las ciencias cercanas con las cuales ella interactúa.

Tal exposición sobre la didáctica de la matemática beneficia al docente de matemáticas en la distinción de los aspectos más importantes en la enseñanza de los contenidos programáticos de la misma, renombrada como cálculo, algebra, lógica, estadística, matemática financiera, entre otras. Dispuestas en el currículo de formación de los aspirantes a una carrera profesional.

\section{Didáctica Crítica de la Matemática Financiera}

En los apartados anteriores se da una breve revisión a los conceptos de educación, educación crítica, didáctica y didáctica de la matemática con la intención de intensificar el carácter social de la educación en la formación de ciudadanos y la importancia de conocer y manejar en el contexto la disciplina matemática, sobre todo cuando se requiere de la enseñanza y aprendizaje de la misma.

En Latinoamérica incluyendo al Ecuador, se sigue aferrado al dominio de la racionalidad técnica instrumental de la matemática por parte del docente en su enseñanza, razón por la cual, 


\section{Didáctica Crítica de la Matemática Financiera}

Vol. 2, núm. 2., (2018)

Shirley Janeth Vélez Alcívar; Eduardo Alfredo Bautista; Jorge Alfredo Espín Martínez

aun predomina la praxis educativa centrada en clases expositivas, logrando en el estudiante un conocimiento poco significativo, al sentir un desfase entre los contenidos y lo que son sus necesidades en cuanto a desarrollar en ellos sus talentos, creatividades y habilidades, para relacionarlos con otras especialidades como la ingeniería, la medicina, la sociología, la psicología, entre otras.

La matemática financiera en este sentido no escapa a esta anuencia, independientemente que la misma se refiera a la matemática aplicada a los negocios, al cálculo mercantil, a su relación con las tasas de interés del sistema financiero, y a los indicadores en la toma de decisiones para inversión, y cálculos de los indicadores de rendimiento.

Insertar la matemática financiera a la didáctica crítica, significa estar al corriente de diversas concepciones sobre la didáctica crítica y la didáctica de la matemática crítica. La didáctica crítica La didáctica crítica surge hacia la mitad del siglo XX como un cuestionamiento a los principios de la escuela tradicional y la didáctica tecnocrática. Se manifiesta por la reflexión colectiva entre docentes y discentes sobre problemas que se encuentran en su propio contexto. Anónimo (p.4) en Bastardo (2016).

La puesta en marcha de la didáctica crítica se basa en estrategias de reflexión, de discusiones y negociación constante que facilitan a los estudiantes acceder a un escenario donde ellos juegan un papel protagónico, al igual que los profesores. La didáctica critica se busca analizar la práctica educativa en donde el aprendizaje sea concebido como un proceso que manifieste constantes momentos de ruptura y reconstrucción, buscando diferenciarse de los planteamientos mecanicistas del aprendizaje, como han sido enfocados desde la didáctica tradicional, la cual se ha centrado más en el resultado que en el proceso. 


\section{Didáctica Crítica de la Matemática Financiera}

Vol. 2, núm. 2., (2018)

Shirley Janeth Vélez Alcívar; Eduardo Alfredo Bautista; Jorge Alfredo Espín Martínez

En este sentido, con la didáctica crítica se busca desvincular la dependencia del docente, el estudiante y la asignatura objeto del conocimiento, con la idea de reconstruir el conocimiento a partir de la reflexión colectiva y la problematización. Así mismo, con la didáctica crítica en la enseñanza de la matemática financiera, se torna como reto, el lograr que la misma no sea algo externa al estudiante, a su experiencia vital, como lo asegura Goñi (2000) en Bastardo (2016), que logremos plantearles problemas que puedan integrarlos en un contexto próximo a ellos, que les permitan desarrollar capacidades realmente útiles fuera de la vida académica" (p.94).

Estos aportes buscan humanizar esta ciencia y debatir nuevos enfoques y aportes teóricos en cuanto a su enseñanza ya que, en la actualidad seguimos encontrándonos con clases de matemática donde los docentes siguen dejando a un lado la discusión de ideas, la comunicación de pensamientos entre éstos y los discentes, de allí la necesidad de incorporar la didáctica crítica en la enseñanza de la matemática.

\section{Consideraciones Finales}

La didáctica crítica ha de ser considerada como un elemento emancipador, dado que la ciencia de la didáctica trata de cambiar la actitud del estudiante como persona individual, a una más activa y cooperativa; y a la actitud del docente, de simple comunicador, a un ente de acción comunicativa en aras de incorporar nuevas bases de integración social a través de vivencias para el logro de una enseñanza más humanizadora del ser.

Además con la didáctica crítica el aprendizaje se concibe como un proceso en equipo y en construcción que se define por los cambios individuales y sociales de esta sociedad cambiante. Tal didáctica en la enseñanza de la matemática financiera, destaca la necesidad de sensibilizar a 


\section{Didáctica Crítica de la Matemática Financiera}

Vol. 2, núm. 2., (2018)

Shirley Janeth Vélez Alcívar; Eduardo Alfredo Bautista; Jorge Alfredo Espín Martínez

los docentes en esta área, en su vocación y dedicación. En pro de ser profesionales reflexivos, profesionales críticos con nuestra práctica, como manera de ir mejorando cada día el quehacer diario en el aula de clases.

\section{Referencias Bibliográficas.}

Alvarado, J. (2012). Pedagogía Crítica y Educación. Disponible en: http://digibug.ugr.es/bitstream/handle/10481/32270/ReiDoCrea-Vol.3-Art.21-

Bastardo, R. (2016). Didáctica crítica en la enseñanza de la matemática desde una visión ética. Revista educare, Vol. 20. Disponible en: http://revistas.upel.edu.ve/index.php/educare/article/viewFile/3468/2716

D’Amore, B. (2006): Didáctica de la matemática. Cooperativa Editorial Magisterio, Universidad de Bogotá. Colombia.

Freire, P. (1978). Pedagogía del Oprimido. Siglo XXI Editores, S.A. Cali-Colombia.

García, S. (2012). Importancia de la matemática financiera. Disponible en: http://reddocente.uladech.edu.pe/forum/topics/importancia-de-la-matematica-financiera

Santaella, E. (2012). Pedagogía crítica, una propuesta educativa para la transformación social. Reidocrea. Volumen 3. Artículo 21. Disponible en: http://digibug.ugr.es/bitstream/handle/10481/32270/ReiDoCrea-Vol.3-Art.21$\underline{\text { Santaella.pdf;jsessionid=289CDAF22C67CBC907CE554CBF1FCCDE? sequence }=1}$

Skovsmose, O. (1999). Hacia una filosofía de la educación matemática crítica. Una empresa docente. Univeridad de Los Andes. Colombia.

Ugas F. G. (2007). Epistemología de la Educación y la Pedagogía. 2a edición. Ediciones del Taller Permanente de Estudios Epistemológicos en Ciencias Sociales. San Cristóbal. Táchira. Venezuela.

Vadillo, G y Klinger, C. (2004): Didáctica. Teoría y Práctica de éxito en Latinoamérica y España. McGraw-Hill / Interaméricana Editores, S. A de C.V. México, D. F.

Valiente, S. (2000): Didáctica de la Matemática: El libro de los recursos. Editorial La Muralla, S.A. Madrid. España. 